\title{
Reasoning with Disjunctive Constrained Tuple-Generating Dependencies ${ }^{\star}$
}

\author{
Junhu Wang ${ }^{1}$, Rodney Topor ${ }^{1}$, and Michael Maher ${ }^{1,2}$ \\ 1 CIT, Griffith University, Brisbane, Australia 4111 \\ jwang, rwt, mjm@cit.gu.edu.au \\ 2 DMCS, Loyola University, Chicago, USA \\ mjm@math.luc.edu
}

\begin{abstract}
In extended relational databases, queries and integrity constraints often contain interpreted variables and built-in relations. We extend previous work on semantic query containment for extended relational databases to handle disjunctive constrained tuple-generating dependencies (DCTGDs) which include almost all well-known classes of integrity constraints. After defining this extended class of integrity constraints, we present a method for expanding a query $Q$, using DCTGDs, to a semantically equivalent set of queries. Our theorems on this method unify and generalize several previous results on semantic query containment. We apply the method to the DCTGD implication problem and prove that, when restricted to regular CTGDs, our method is strictly more powerful than previously published chase algorithms.
\end{abstract}

\section{Introduction}

Modern relational database systems storing complex data such as spatial, audio, image, video or temporal data need the ability to store and manipulate built-in relations in addition to database relations. In conventional databases, examples of such built-in relations include arithmetic relations such as $X<Y$ or $Z=X+$ $Y$. In spatial or image databases, examples include topological and directional relations between spatial objects, such as $X$ contains $Y$ or $X$ is north of $Y$. In temporal and video databases, examples include temporal relations and kinetic properties. In many cases, evaluation of queries involving built-in relations is highly expensive, computationally, but built-in relations are necessary to achieve a required level of expressiveness. We will adopt the terminology of constraint databases, and refer to built-in relations as constraints.

The data in such database systems is also subject to varied applicationspecific semantic restrictions which, in traditional database systems, are represented as integrity constraints. One of the widest class of integrity constraints is the constrained tuple-generating dependencies (CTGDs)[1], which generalize traditional dependencies such as FDs, MVDs, EGDs, TGDs[2] and CGDs[3]. Here, we further extend this class to allow disjunctions. We call the resulting

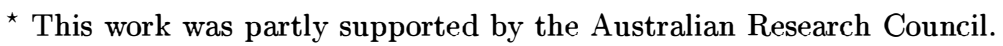


class of integrity constraints disjunctive constrained tuple-generating dependencies (DCTGDs). Such dependencies are needed to represent a wide array of common semantic relationships. For example, an integrity constraint might state that any student is either male or female:

$$
\operatorname{student}(x) \rightarrow \text { male }(x) \vee \text { female }(x)
$$

More generally, whenever a class is partitioned into subclassess there is an implicit disjunctive integrity constraint. Disjunctive restrictions may also arise directly from the semantics of the data. For example, an integrity constraint might state that a road crosses a river either over a bridge or through a tunnel:

$$
\begin{aligned}
\operatorname{road}(r d i d, & r d \text { geo }), \text { river }(r v i d, r v \text { geo }), r d \text { geo } \cap \text { rv geo } \neq \varnothing \rightarrow \\
& (\exists b \text { id, } b \text { geo bridge }(b \text { id, } b \text { geo }), \text { b geo } \cap \text { rd geo } \cap \text { rv geo } \neq \varnothing) \vee \\
& (\exists t \text { id,t geo tunnel }(t \text { id, } t \text { geo }), t \text { geo } \cap r d \text { geo } \cap \text { rv geo } \neq \varnothing) .
\end{aligned}
$$

As argued in [4], sophisticated query optimization which incorporates understanding of the built-in relations and exploitation of highly expressive integrity constraints will be cost-effective for modern applications where the potential for improvement is much greater than in traditional databases. Two key problems are testing query containment and testing integrity constraint implication. Previous researchers have developed and studied techniques for proving implication of integrity constraints $[1,3,5,6]$, and have proposed techniques for query optimization $[4,7]$.

We extend this previous work to handle DCTGDs as follows. In Section 2, we define our generalized classes of integrity constraints and queries. In Section 3, we present a procedure for expanding a query $Q$ in the presence of a set of DCTGDs $\triangle$ into a semantically equivalent set $S$ of queries. We show that, when this procedure terminates, $Q$ is contained in another query $Q^{\prime}$ under $\triangle$ iff every query in $S$ is contained in $Q^{\prime}$ independently of $\triangle$. This effectively reduces a semantic containment problem to a set of standard query containment problems, which can be tested using standard methods such as the above generalization of the containment mapping theorem. We also note the simple conditions under which the query expansion procedure is guaranteed to terminate and other useful properties. When the second query $Q^{\prime}$ is known in advance, we show that a more efficient query expansion procedure can be used to prove $Q$ is semantically contained in $Q^{\prime}$ under $\triangle$. This is done in Section 4. In Section 5, we apply our query expansion procedure to the the implication problem for DCTGDs. We show our procedure is strictly more powerful than the chase algorithms of [1]. Section 6 concludes the paper with a comparison with related work. We show that our theorems unify and generalize several results in [6] and [4]. Furthermore, the technique of expansion is substantially simpler than the techniques used in those previous works, so that we achieve a conceptual simplification in addition to unifying results. 


\section{Preliminaries}

In this section, we review the terminology in extended relational databases and introduce definitions and notations which will be used throughout the paper. In particular, we introduce DCTGDs which extend CTGDs[1].

A term is a variable, or constant, or function over terms. An atom is a formula of the form $r\left(t_{1}, \ldots, t_{n}\right)$ where $r$ is an uninterpreted predicate (i.e, a database relation name) and $t_{1}, \ldots, t_{n}$ are terms ${ }^{1}$. In this paper, a conjunction of atoms $r_{1} \wedge \cdots \wedge r_{n}$ is also written as $r_{1}, \ldots, r_{n}$ for short, and is often treated as a set $\left\{r_{1}, \ldots, r_{n}\right\}$. A finite conjunction of atoms is said to be normalized if the terms in each atom are distinct variables, and the variables in different atoms are disjoint. For instance, the conjunction $r_{1}(x, y), r_{2}(u, v)$ is normalized while $r_{1}(x, y), r_{2}(y, z)$ and $r_{1}(x, 1)$ are not.

A primitive constraint over domain $\mathcal{D}$ is a formula of the form $c\left(x_{1}, \ldots, x_{n}\right)$ where $c$ is a system built-in, interpreted predicate, $x_{1}, \ldots, x_{n}$ are variables over $\mathcal{D}$, i.e, $c$ defines a n-ary built-in relation which, given an assignment of elements from $\mathcal{D}$ to the variables, can be evaluated to TRUE or FALSE by the system. A constraint is a formula obtained by a combination of the following operations on primitive constraints: renaming, quantification, logical negation, conjunction and disjunction.

If $R$ is a set of atoms, then $\operatorname{Var}(R)$ and $\operatorname{Term}(R)$ will denote the set of variables and the set of terms, respectively, in $R$. If $C$ is a constraint, then $\operatorname{Var}(C)$ will denote the set of free variables in $C$, and $C$ will denote the logical negation of $C$.

\subsection{Disjunctive Constrained Tuple-Generating Dependencies}

Definition 1. $A$ disjunctive constrained-tuple generating dependency (DCTGD) is a formula of the form

$$
P, C \rightarrow \bigvee_{i=1}^{k}\left(\exists Y_{i} P_{i}^{\prime}, C_{i}^{\prime}\right)
$$

where $P$ and $P_{i}^{\prime}$ are conjunctions of atoms, $C$ and $C_{i}^{\prime}$ are constraints, $Y_{i}$ is a set of variables disjoint from $\operatorname{Var}(P), \operatorname{Var}\left(P_{i}^{\prime}\right) \subseteq \operatorname{Var}(P) \cup \operatorname{Var}(C) \cup Y_{i}$ and $\operatorname{Var}\left(C_{i}^{\prime}\right) \subseteq \operatorname{Var}(P) \cup \operatorname{Var}(C) \cup Y_{i}$ for $i=1, \ldots, k$. If $P$ contains at least one atom and $\operatorname{Var}(C) \subseteq \operatorname{Var}(P)$, we say the DCTGD is regular ${ }^{2}$.

In a DCTGD, the left-hand side of the arrow is referred to as the antecedent and the right-hand side is referred to as the consequent. For convenience, we will assume that the set of atoms in the antecedent is normalized (although we will use more compact forms in some examples). When there is only one disjunct in

${ }^{1}$ In this paper, the functions are assumed to have fixed interpretations which the system understands.

2 Note that most practical DCTGDs are regular. 
the consequent (i.e, $k=1$ in the above definition) and the constraints in either side of the arrow is a conjunction of primitive constraints, the DCTGD becomes a constrained tuple-generating dependency (CTGD)[1].

\subsection{General Conjunctive Queries}

Definition 2. A general conjunctive query (GCQ) is a formula of the form

$$
\{V: R, D\}
$$

where $V=\left(v_{1}, \ldots, v_{k}\right)$ is a sequence of variables, $R$ is a conjunction of atoms, $D$ is a constraint, and $\operatorname{Var}(D) \subseteq V \cup \operatorname{Var}(R)$. We call $V$ and $R, D$ the output and the body of the query, respectively.

Note that the conventional conjunctive query [5] is a GCQ in which the constraint in the body is a conjunction of equalities $(=)$, disequalities $(\neq)$, and (if the domains are ordered) comparisons such as $<$ and $\leq$. Note also that $V$ is a sequence. But for convenience we sometimes treat it as a set.

For any query $Q$, the answer set $Q(\mathcal{I})$ of $Q$ with respect to a database instance $\mathcal{I}$ is the set of all answers to $Q$ with respect to $\mathcal{I}$. A query $Q$ is said to be contained in another $Q^{\prime}$, denoted $Q \sqsubseteq Q^{\prime}$, if $Q(\mathcal{I}) \subseteq Q^{\prime}(\mathcal{I})$ for every instance $\mathcal{I}$. $Q$ and $Q^{\prime}$ are said to be equivalent, denoted $Q=Q^{\prime}$, if $Q \sqsubseteq Q^{\prime}$ and $Q^{\prime} \sqsubseteq Q$. $Q$ is said to be empty, if $Q(\mathcal{I})=\varnothing$ for every instance $\mathcal{I}$. Clearly, if $Q$ is a GCQ, then $Q$ is empty if and only if the constraint in its body is unsatisfiable.

The above concepts of query containment and equivalence extend to semantic containment and equivalence if we are interested only in instances that satisfy a set of DCTGDs.

Definition 3. Let $\triangle$ be a set of DCTGDs, and $Q_{1}$ and $Q_{2}$ be queries. If for any instance $\mathcal{I}$ satisfying $\triangle$, we have $Q_{1}(\mathcal{I}) \subseteq Q_{2}(\mathcal{I})$, then we say that $Q_{1}$ is (semantically) contained in $Q_{2}$ under $\triangle$, denoted as $Q_{1} \sqsubseteq \triangle Q_{2}$. If $Q_{1} \sqsubseteq \triangle Q_{2}$ and $Q_{1} \sqsubseteq \triangle Q_{2}$, then we say that $Q_{1}$ and $Q_{2}$ are equivalent under $\triangle$, denoted as $Q_{1}=\triangle Q_{2}$.

In the sequel, we will assume all of the GCQs are safe as defined below.

Definition 4. A GCQ $\{V: R, D\}$ is said to be safe if

(1) $R$ contains at least one atom, and

(2) Every output variable either appears in $\operatorname{Var}(R)$, or is equated to a (interpreted) function of $\operatorname{Var}(R)$ or to a constant by $D$.

For convenience, we sometimes require the GCQs be in normal form.

Definition 5. A GCQ $\{V: R, D\}$ is said to be in normal form (NF) if $R$ is normalized, the variables in the sequence $V$ are distinct, and $V$ and $\operatorname{Var}(R)$ are disjoint.

Clearly every GCQ has an equivalent GCQ which is in NF. 


\subsection{Containment Mappings}

We need two types of containment mappings. The first type is from a regular DCTGD to a GCQ, and is used mainly in expanding GCQs; the second type is from one GCQ to another, and is used in testing containment.

Definition 6. Let $f \equiv P, C \rightarrow \bigvee_{i=1}^{k}\left(\exists Y_{i} P_{i}^{\prime}, C_{i}^{\prime}\right)$ be a regular DCTGD, and $Q \equiv\{V: R, D\}$ be a GCQ. A containment mapping (CM) from $f$ to $Q$ is a mapping from $\operatorname{Var}(P)$ to $\operatorname{Term}(R)$ such that it maps every atom in $P$ to an atom in $R$.

Definition 7. Let $Q_{i} \equiv\left\{V_{i}: R_{i}, D_{i}\right\} \quad(i=1,2)$ be two GCQs. A containment mapping $(\mathrm{CM})$ from $Q_{2}$ to $Q_{1}$ is a mapping from $V_{2} \cup \operatorname{Var}\left(R_{2}\right)$ to $V_{1} \cup \operatorname{Term}\left(R_{1}\right)$ such that it maps the sequence $V_{2}$ to the sequence $V_{1}$, and maps every atom in $R_{2}$ to some atom in $R_{1}$.

Let $\delta$ be a CM defined on variables $x_{1}, \ldots, x_{n}$. Given any set of atoms and/or constraints $\mathcal{H}, \delta(\mathcal{H})$ denotes the set of atoms and/or constraints that is obtained by simultaneously replacing each occurrence of the variables $x_{i}$ in $\mathcal{H}$ by $\delta\left(x_{i}\right)$. In addition, two CMs from the same DCTGD (to possibly different GCQs) are considered to be the same if they map every variable to the same term.

The following lemma is straightforward.

Lemma 1. Let $Q \equiv\{V: R, D\}$ be a $G C Q$, and $f \equiv P, C \rightarrow \bigvee_{i=1}^{k}\left(\exists Y_{i} P_{i}^{\prime}, C_{i}^{\prime}\right)$ be a regular DCTGD. If $\delta$ is an $C M$ from $f$ to $Q$, then $Q$ is equivalent under $f$ to the union of

$$
\begin{aligned}
& Q_{0}=\{V: R, D \wedge \delta(C)\} \text { and } \\
& Q_{i}=\left\{V: R, \delta\left(P_{i}^{\prime}\right), D \wedge \delta\left(C \wedge C_{i}^{\prime}\right)\right\}(i=1, \ldots, k),
\end{aligned}
$$

where the variables $Y_{i}$ are renamed to distinct variables not in $V \cup \operatorname{Var}(R)$.

The next lemma is slightly generalized from [8]. It can be used as a basic tool for detecting GCQ containment and equivalence.

Lemma 2. Let $Q_{i} \equiv\left\{V_{i}: R_{i}, D_{i}\right\} \quad(i=0,1,2, \ldots, n)$ be GCQs. Suppose $Q_{0} \neq \varnothing$, and $Q_{1}, \ldots, Q_{n}$ are in $N F$. Then $Q_{0} \sqsubseteq \bigcup_{i=1}^{n} Q_{i}$ iff there are $C M s \delta_{i, 1}, \ldots, \delta_{i, k_{i}}$ from $Q_{i}$ to $Q_{0}$ such that $D_{0} \rightarrow \vee_{i=1}^{n} \vee_{j=1}^{k_{i}} \delta_{i, j}\left(D_{i}\right)$.

\section{Semantic Expansion}

\subsection{The Procedure Expand}

Before formally defining the procedure, let us look at the intuition first.

Let $\triangle$ be a set of regular DCTGDs and $Q \equiv\{V: R, D\}$ be a GCQ. As shown in Lemma 1 , if there is a DCTGD $f_{1} \equiv P, C \rightarrow \bigvee_{i=1}^{k}\left(\exists Y_{i} P_{i}^{\prime}, C_{i}^{\prime}\right)$ in $\triangle$ and an CM $\delta$ from $f_{1}$ to $Q$, then $Q$ is equivalent under $f_{1}$ to the union of

$Q_{0} \equiv\{V: R, D \wedge \delta(C)\}$ and

$Q_{i} \equiv\left\{V: R, \delta\left(P_{i}^{\prime}\right), D \wedge \delta\left(C \wedge C_{i}^{\prime}\right)\right\}(i=1, \ldots, k)$,

where the variables in $Y_{i}$ are renamed to distinct new variables not in $\operatorname{Var}(R) \cup V$. 
To each of $Q_{0}, Q_{1}, \ldots, Q_{k}$, if there is a CM from $f_{2} \in \triangle$, then it can be split into the union of some other GCQs in a similar way, and thus $Q$ will be equivalent under $f_{1}$ and $f_{2}$ to the union of more GCQs. Continuing this process, we can split $Q$ into the union of more and more GCQs under $\triangle$. In general, regarding the GCQs in the union as a set $S$, and continuing as above, we hope to be able to reach a point where no matter what DCTGD in $\triangle$ and what mapping we use, we can not split any GCQ in $S$ into more GCQs which are not subsumed by other GCQs in $S$.

Clearly the splitting of a GCQ $Q^{\prime}$ into $Q_{0}^{\prime}, \ldots, Q_{k}^{\prime}$ in the above process depends on the application of a (DCTGD, CM) pair. Let us call $Q_{0}^{\prime}, \ldots, Q_{k}^{\prime}$ the child queries of $Q^{\prime}$, and use the name descendant (query) for a GCQ's child query or its descendant's child query. For efficiency, any (DCTGD, CM) pairs, if already applied in splitting $Q^{\prime}$, should not be used again to split $Q^{\prime}$ 's descendants. Also, any newly generated GCQs in $S$ which are empty can be removed immediately.

We now formalize the above idea into the procedure Expand as listed in Fig 1.

\section{Procedure Expand $(Q, \triangle)$}

Input: a GCQ $Q$ and a set $\triangle$ of regular DCTGDs.

Output: a set of GCQs $S$.

Local variables: a set pairs $\left(Q^{\prime}\right)$ for each GCQ $Q^{\prime}$ in $S$.

let $S=\emptyset$; let pairs $(Q)=\emptyset$;

if $(Q$ is empty)

then return $S$;

else add $Q$ to $S$;

while (there is $Q^{\prime} \equiv\{V: R, D\}$ in $S$ and $f \equiv P, C \rightarrow \bigvee_{i=1}^{k}\left(\exists Y_{i} P_{i}^{\prime}, C_{i}^{\prime}\right)$ in $\triangle$, and an CM $\delta$ from $f$ to $Q^{\prime}$ such that $(f, \delta) \notin$ pairs $\left.\left(Q^{\prime}\right)\right)$ do

let $Q_{0}=\{V: R, D \wedge \delta(C)\}$;

$Q_{i}=\left\{V: R, \delta\left(P_{i}^{\prime}\right), D \wedge \delta\left(C \wedge C_{i}^{\prime}\right)\right\}(i=1, \ldots, k)$

where the variables in $Y_{i}$ are renamed to distinct variables not in $\operatorname{Var}(R) \cup V$.

if (no $Q_{i}(i=0, \ldots, k)$ is equivalent to $\left.Q^{\prime}\right)$

then remove $Q^{\prime}$ from $S$;

for $(i=0, \ldots, k)$ do

if $\left(Q_{i}\right.$ is not empty )

then add $Q_{i}$ to $S$; let pairs $\left(Q_{i}\right)=$ pairs $\left(Q^{\prime}\right) \cup\{(f, \delta)\}$;

else let pairs $\left(Q^{\prime}\right)=\operatorname{pairs}\left(Q^{\prime}\right) \cup\{(f, \delta)\}$;

return $S$;

Fig. 1. The semantic expansion procedure

Procedure Expand $(Q, \triangle)$ may or may not terminate. If it does, we will have a finite set $S$ of GCQs . Let $Q^{\triangle}$ be the union of these GCQs if $S \neq \varnothing$, and let $Q^{\triangle}$ be an empty query if $S=\emptyset$. It is easy to see that $Q^{\triangle}=\triangle Q$.

Let us call the process defined by the procedure $\operatorname{Expand}(Q, \triangle)$ the semantic expansion of query $Q$ with $\triangle$. Using semantic expansion, query containment under DCTGDs can be reduced to query containment without DCTGDs. 
Theorem 1. Given a set of regular DCTGDs $\triangle$ and a GCQ $Q$, if the procedure $\operatorname{Expand}(Q, \triangle)$ terminates with result $S$, then for any query $Q^{\prime}, Q \sqsubseteq \triangle Q^{\prime}$ if and only if every GCQ in $S$ is contained in $Q^{\prime}$ (without the DCTGDs).

Note that $Q^{\prime}$ can be any query, including unions and differences of GCQs. In particular, when $Q^{\prime}$ is the empty query, we get the following corollary.

Corollary 1. Let $Q$ be $a G C Q$ and $\triangle$ be a set of regular DCTGDs. If the procedure Expand $(Q, \triangle)$ terminates with result $S$, then $Q$ is empty under $\triangle$ iff $S=\varnothing$.

Note also that we have assumed the conjunctions of atoms in the antecendents of the DCTGDs are normalized; if not, the above theorem will not hold.

Example 1. Let $\triangle$ contain the integrity constraint $f \equiv p(x, y), q(y, z) \rightarrow F A L S E$. Let $Q=\{x: p(x, y), q(z, u), y=z\}$. Since there are no CMs from $f$ to $Q$, Expand terminates with $S=\{Q\}$, which is not empty, but $Q$ is empty under $f$.

When the DCTGDs are limited to disjunctive TGDs, i.e, DCTGDs that do not have constraints in the consequent, we get the next corollary.

Corollary 2. Let $\triangle$ be a set of regular disjunctive TGDs, and $Q$ be a non-empty $G C Q$. If the procedure Expand $(Q, \triangle)$ terminates, then $Q$ is not empty under $\triangle$.

\subsection{Termination and Other Properties}

Given that Theorem 1 holds on the condition that Expand terminates, it is natural to ask when Expand will terminate. In general, the termination problem is undecidable. However, for some special classes of DCTGDs, termination of Expand is guaranteed. In this section, we identify some of these special classes.

First, it is trivial to see that when $\triangle$ is a set of (disjunctive) CGDs, the expansion terminates for any GCQ $Q$. The only possible non-terminating case is when there are DCTGDs that contain atoms in the consequent.

Definition 8. A set of DCTGDs $\triangle$ is said to be recursive, if it contains a sequence of DCTGDs $f_{1}, \ldots, f_{n}(n \geq 1)$ such that some relation names in the antecedent of $f_{i+1}$ appear in the consequent of $f_{i}$ (for $\left.i=1, \ldots, n-1\right)$, and some relation names in the antecedent of $f_{1}$ appear in the consequent of $f_{n}$.

Proposition 1. If the regular DCTGDs in $\triangle$ are not recursive, then Expand $(Q, \triangle)$ terminates for any $G C Q Q$.

Even for recursive DCTGDs, there are simple conditions under which Expand terminates on any GCQ $Q$.

Definition 9. A regular DCTGD $P, C \rightarrow \bigvee_{i=1}^{k} \exists Y_{i} P_{i}^{\prime}, C_{i}^{\prime}$ is said to be a full dependency if $\operatorname{Var}\left(P_{i}^{\prime}\right) \subseteq \operatorname{Var}(P)$ for $i=1, \ldots, k$. 
Proposition 2. If $\triangle$ is a set of full dependencies, and the atoms in the consequents of these dependencies do not contain functions, then the procedure Expand $(Q, \triangle)$ terminates for any $G C Q Q$.

The condition that there are no functions is necessary.

Example 2. If $\triangle$ contains $p(x, y) \rightarrow p(x, y+1)$ only ${ }^{3}$, and $Q=\{x: p(x, y)\}$, where $x, y$ are from the reals, then the body of $Q$ can be expanded to $p(x, y), p(x, y+$ $1), p(x, y+2), \ldots$, so the procedure $\operatorname{Expand}(Q, \triangle)$ will not terminate.

Note that in Proposition 1 and Proposition 2, we have implicitly assumed there are terminating algorithms for solving implication problems of the underlying constraints.

There are many other cases where the semantic expansion procedure terminates. In general, the termination also depends on the query $Q$. A trivial case of termination, for example, is when there are no CMs from any DCTGDs to $Q$. The order of choosing a DCTGD from $\triangle$ may also affect termination. For example, if $\triangle$ contains $p(x, y), x>y \rightarrow F A L S E$ and $p(x, y) \rightarrow \exists z p(x, z), z>y$, and $Q=\{x: p(x, y), x>y\}$, then Expand will terminate immediately if we choose the first DCTGD, but it will not terminate if we always give priority to the second DCTGD.

In practical applications of Theorem 1 (e.g, when testing semantic query containment), we can add extra conditions in the procedure so that it may terminate earlier, as will be seen in Section 4 .

In addition, we note the following properties of Expand:

1. Although, in general, the number of GCQs in $S$ grows exponentially, there are useful special cases where there will be at most one GCQ in the final $S$. For example, when there are only CTGDs in $\triangle$ and there is no constraint in the antecedent of any CTGD, or when there are only dependencies of the form $P, C \rightarrow F A L S E$.

2. In Expand, if a (DCTGD, CM) pair is applicable to a GCQ $Q^{\prime}$ in $S$ but not applied, then it will remain applicable to $Q^{\prime}$ 's descendants (if the descendants are added to $S$ ) until it is applied. Thus different orders of choosing a DCTGD or CM will not affect the final result if they all lead to termination of the procedure.

3. There are several variations of Expand for which Theorem 1 remains valid. For instance, we can replace " $Q_{i}=\left\{V: R, \delta\left(P_{i}^{\prime}\right), D \wedge \delta\left(C \wedge C_{i}^{\prime}\right)\right\}$ " with " $Q_{i}=\left\{V: R, \delta\left(P_{i}^{\prime}\right), D \wedge \delta\left(C_{i}^{\prime}\right)\right\}$ ". We can also relax the condition "if $\left(Q_{i}\right.$ is not empty)" or replace it with "if $\left(Q_{i}\right.$ is not contained in the union of the GCQs in $S)$ ". The condition "if (no $Q_{i}(i=0, \ldots, k)$ is equivalent to $\left.Q^{\prime}\right)$ " can also be relaxed. The differences lie in the efficiency of the procedure.

4. Procedure Expand and Theorem 1 extend naturally to the case where $Q$ is a union of GCQs. We only need to modify Expand so that every GCQ in the union is added to $S$ before applying the DCTGDs.

${ }^{3}$ Our assumption that the DCTGD is regular and normalized disallows rewriting this integrity constraint to $p(x, z), z=y-1 \rightarrow p(x, y)$ or $p(x, y-1) \rightarrow p(x, y)$. 


\section{Application to Testing Semantic Query Containment}

Obviously, Theorem 1 can be used to test whether $Q \sqsubseteq \triangle Q^{\prime \prime}$ for GCQ $Q$ and an arbitrary query $Q^{\prime \prime}$ (provided there are methods to test whether a GCQ is contained in $\left.Q^{\prime \prime}\right)$. In fact, if $Q^{\prime \prime}$ is given in advance, it is possible that $Q \sqsubseteq \triangle Q^{\prime \prime}$ or $Q \nsubseteq \triangle Q^{\prime \prime}$ be detected before Expand terminates. The procedure Ctest listed in Fig. 2 is modified from Expand to accommodate $Q^{\prime \prime}$.

\section{Procedure $\operatorname{Ctest}\left(Q, \triangle, Q^{\prime \prime}\right)$}

Input: a GCQ $Q$, a set $\triangle$ of regular DCTGDs, and an arbitrary query $Q^{\prime \prime}$. Output: TRUE or FALSE.

Local variables: a set pairs $\left(Q^{\prime}\right)$ for each GCQ $Q^{\prime}$ in $S$.

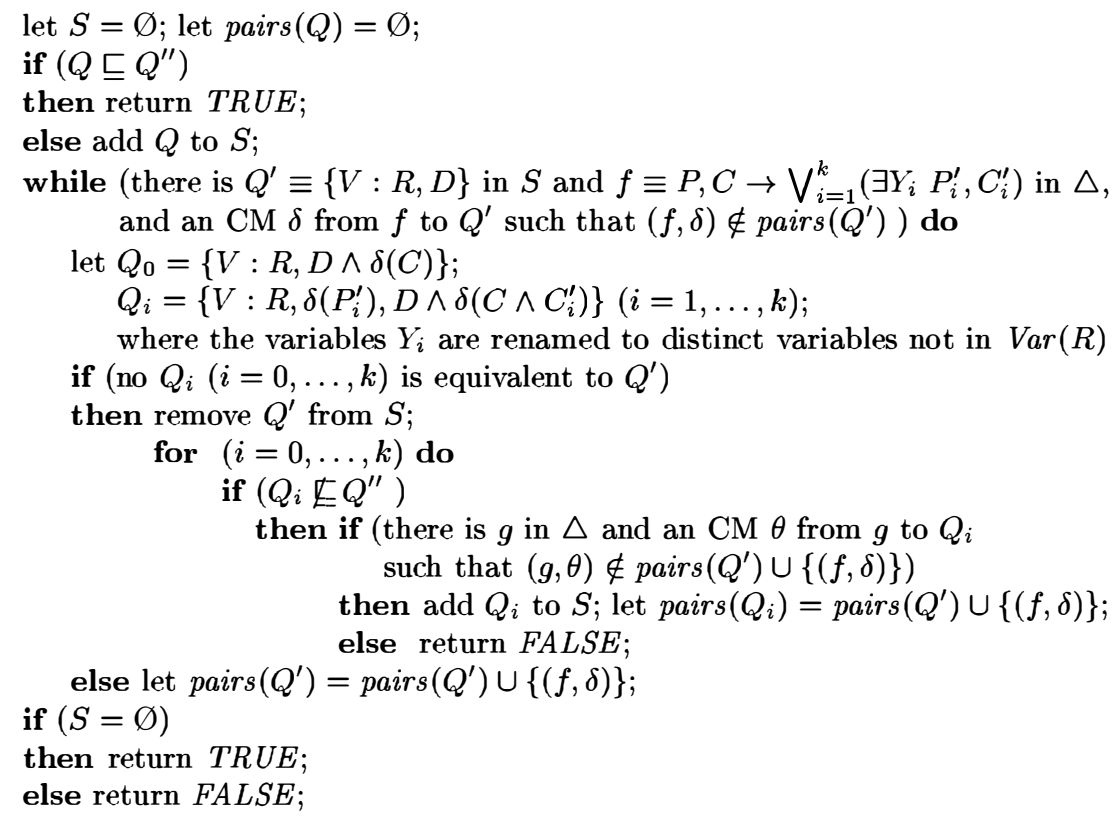

Fig. 2. The procedure for semantic query containment

Theorem 2. Let $Q$ be a GCQ and $\triangle$ be a set of regular DCTGDs. Let $Q^{\prime \prime}$ be any query. If Ctest $\left(Q, \triangle, Q^{\prime \prime}\right)$ returns TRUE, then $Q \sqsubseteq \triangle Q^{\prime \prime}$. If Ctest $\left(Q, \triangle, Q^{\prime \prime}\right)$ returns FALSE, then $Q \nsubseteq \triangle Q^{\prime \prime}$.

The next two examples demonstrate the application of Ctest. In Example 3, Ctest detects $Q \nsubseteq \triangle Q^{\prime \prime}$, but Expand does not terminate. In Example 4, Ctest detects $Q \sqsubseteq \triangle Q^{\prime \prime}$, but, again, $\operatorname{Expand}(Q, \triangle)$ does not terminate.

Example 3. Let $Q=\{x: p(x)\}, Q^{\prime \prime}=\{x: p(x), q(x, y)\}$. Let $\triangle=\left\{f_{1}, f_{2}\right\}$, where $f_{1}, f_{2}$ are $p(x), x>0 \rightarrow \exists y q(x, y)$ and $q(x, y), y>0 \rightarrow p(y)$ repectively. Applying $f_{1}$ to $Q$, we get a GCQ $Q_{1} \equiv\{x: p(x), x \leq 0\}$ which is not contained 
in $Q^{\prime \prime}$. Since the only $\mathrm{CM}$ from $f_{1}$ to $Q_{1}$ has been used, and there is no CM from $f_{2}$ to $Q_{1}, C$ test returns FALSE. So we know $Q \nsubseteq \triangle Q^{\prime \prime}$.

Example 4. Let $Q=\{y: p(x, y), q(y), y<0\}$ and $Q^{\prime \prime}=\{y: p(x, y), q(y), y<$ $0, x \leq 0\}$. Let $\Delta=\left\{f_{1}, f_{2}, f_{3}\right\}$ where $f_{1}, f_{2}$, and $f_{3}$ are $p(x, y), x>0 \rightarrow r(x, y)$; $r(x, y), q(y), z=y, \rightarrow x<-10 ;$ and $q(y), y<0 \rightarrow \exists z p(y, z)$ respectively. Applying $\left(f_{1}, I\right)$ ( $I$ is the identity mapping) to $Q$, we split $Q$ to

$$
Q_{1} \equiv\{y: p(x, y), q(y), y<0, x \leq 0\} \text { and }
$$

$$
Q_{2} \equiv\{y: p(x, y), q(y), r(x, y), y<0, x>0\}
$$

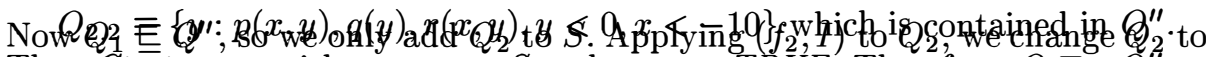
Thus Ctest stops with an empty $S$ and returns TRUE. Therefore, $Q \sqsubseteq \triangle Q^{\prime \prime}$.

\section{Application to the DCTGD Implication Problem}

In this section, we implicitly assume all the DCTGDs are regular.

The DCTGD implication problem is as follows: Given a set $\triangle$ of DCTGDs and a single DCTGD $g$, decide whether $\triangle \models g$, i.e, whether any database instance that satisfies $\triangle$ must also satisfy $g$.

The DCTGD implication problem can be reduced to the semantic containment problem.

Proposition 3. Let $\triangle$ be a set of DCTGDs, and $g$ be $P, C \rightarrow \bigvee_{i=1}^{k}\left(\exists Y_{i} P_{i}^{\prime}, C_{i}^{\prime}\right)$. Then $\triangle \vDash g$ iff $Q \sqsubseteq \triangle Q^{\prime \prime}$, where $Q=\{X: P, C\}$ and $Q^{\prime \prime}=\bigcup_{i=1}^{k}\{X$ : $\left.P, P_{i}^{\prime}, C \wedge C_{i}^{\prime}\right\}(X$ is a sequence of all of the variables in $\operatorname{Var}(P))$.

Thus the procedure Ctest provides a method for the DCTGD implication problem.

[1] gives two chase procedures, Chase1 and Chase2 for the CTGD implication problem using the concept of constrained-tuples. We claim that, when restricted to regular CTGDs, Ctest is more powerful than Chase1 and Chase2 in the sense that any implication or non-implication that can be detected by Chase1 or Chase 2 can also be detected by $C t e s t$, and that Ctest can detect more cases where $\triangle$ does not imply $g$. In addition, Ctest is conceptually simpler.

Theorem 3. Let $\triangle$ be a set of CTGDs, and $g$ be a single CTGD. If $\triangle \not \neq g$ or $\triangle \models g$ can be detected by Chase1 or Chase2, then it can also be detected by Ctest.

Example 5 below demonstrates Ctest is strictly more powerful than the chase procedures in detecting non-implication.

Example 5. Let $\triangle$ be the same set as in Example 3. Let $g$ be $p(x) \rightarrow \exists y q(x, y)$. As shown in Example 3, Ctest can detect that $\{x: p(x)\} \mathbb{E} \triangle\{x: p(x), q(x, y)\}$, thus we know $\triangle \not \# g$. But Chase2 will not terminate and thus can not detect this. Chase1 can not detect this either, because the constraints do not have the INC property[8]. 


\section{Comparison with Related Work}

We have shown in the previous section that Ctest is strictly more powerful than the chase algorithms in [1]. Two other most closely related papers are [6] and [4]. The former studies conjunctive query containment under implication constraints and referential constraints, which are both special CTGDs. A main section of [4] is devoted to query containment under CTGDs and gives a necessary condition in the general case and some necessary and sufficient conditions for several special cases. We claim that Theorem 1 unifies and generalizes all the related results in [6] and [4]. In particular, the theorems 2,3,4 in [4] and the theorems 3.1, 4.1 in [6] are all immediate corollaries of Theorem 1 and Lemma 2. Take the main result of [6] as an example. It says

Corollary 3 (Theorem 3.1 of [6]). Given a set of implication constraints

$N=\left\{i c_{i} \equiv P_{i}, C_{i} \rightarrow F A L S E \mid i=1, \cdots, n\right\}$, and a non-recursive set of referential constraints (TGDs with one atom on each side of the arrow and no built-in constraints) $M, a$ (non-empty) conjunctive query $Q=\{V: R, D\}$ is empty un$\operatorname{der} N \cup M$ if and only if there are CMs $\rho_{i, 1}, \cdots, \rho_{i, n_{i}}$ from ic $i_{i}$ to $Q^{M}$ such that $D \rightarrow \vee_{i=1}^{n} \vee_{j=1}^{n_{i}} \rho_{i, j}\left(C_{i}\right)$.

To derive this corollary, we first note that $\operatorname{Expand}(Q, M)$ generates a single conjunctive query $Q^{M}=\left\{V: R, R^{\prime}, D\right\}$ where $R^{\prime}$ is the set of the newly added atoms. If there are no CMs from any of the integrity constraints in $N$ to $Q^{M}$, then $Q^{M \cup N}=Q^{M}$, by Corollary 2, $Q$ will not be empty under $M \cup N$. Now suppose $\rho_{i, 1}, \cdots, \rho_{i, n_{i}}$ are all the CMs from $i c_{i}$ to $Q^{M}$, then $Q^{M \cup N}=\left(Q^{M}\right)^{N}$ will consist of a single CQ (suppose we use a variation of Expand where there is no emptiness test) $\left\{V: R, R^{\prime}, V, D \wedge \wedge_{i=1}^{n} \vee_{j=1}^{n_{i}} \delta_{i, j}\left(C_{i}\right)\right\}$. Thus, by Corollary 1, $Q$ is empty under $M \cup N$ if and only if $D \wedge \wedge_{i=1}^{n} \wedge_{j=1}^{n_{i}} \delta_{i, j}\left(C_{i}\right)$ is unsatisfiable, that is, $D \rightarrow \vee_{i=1}^{n} \vee_{j=1}^{n_{i}} \delta_{i, j}\left(C_{i}\right)$.

\section{References}

1. M. J. Maher and D. Srivastava. Chasing constrained tuple-generating dependencies. In Proc. ACM Symp. PODS, New York, 1996.

2. R. Fagin and M. Y. Vardi. The theory of data dependencies-an overview. In $L N C S$ v172,1-22, Springer-Verlag, 1984.

3. M. Baudinet, J. Chomicki, and P. Wolper. Constraint-generating dependencies. Journal of Computer and System Sciences 59, 94-115, 1999.

4. M. J. Maher and J. Wang. Optimizing queries in extended relational databases. In DEXA2000, Lodon, LNCS1873, p386-396.

5. J. D. Ullman. Principles of Database and Knowledge-Base Systems, volume 1 \& 2 . Computer Science Press, 1st edition, 1988.

6. X. Zhang and Z. M. Özsoyoglu. Implication and referential constraints: A new formal reasoning. IEEE TKDE, 9(6):894-910, Nov/Dec 1997.

7. S. Chaudhuri and K. Shim. Query optimization in the presence of foreign functions. In Proc. of the 19th international conference on VLDB, Dublin, Ireland, 1993.

8. M. J. Maher. A logic programming view of CLP. In Proc. 10th International Conference on Logic Programming, pages 737-753, Budapest, Hungary, 1993. 